\title{
Ventilatory support recommendations in children with Sars-CoV-2
}

Werther Brunow de Carvalho'

(iD) Isadora Souza Rodriguez ${ }^{1}$

Emiliana Holzhausen Gonçalves da Motta ${ }^{1}$

(iD) Artur Figueiredo Delgado ${ }^{1}$

1. Instituto da Criança do Hospital das Clínicas da Faculdade de Medicina da Universidade de São Paulo

KEYWORDS: Sars-CoV-2, critically ill patients, mechanical ventilation, respiratory support, children, adolescents

\section{INTRODUCTION}

COVID-19 is a new respiratory illness that can rapidly deteriorate into severe respiratory distress. Since a specific antiviral treatment is still under investigation, the main way to reduce morbidity and mortality is early and strong intervention when it comes to respiratory support ${ }^{1}$. Initially thought to have flu-like symptoms, this disease has shown that it can present itself as severe pneumonia with a non-uniform presentation: in some cases with preserved compliance and altered perfusion, and sometimes with very low compliance, similarly to acute respiratory distress syndrome.

Recently, one study from Gattinoni et al.2 describes two types of lung lesions: type L and type H. Type L (Fig. 1) patients may have subpleural interstitial edema (ground-glass injuries), low elastance (and high compliance), low ventilation/perfusion ratio (V/Q) - gas volume is nearly normal, and what leads to hypoxemia is altered perfusion and loss of hypoxic vasoconstriction and they also have low lung recruitability; this kind of pattern usually appears at the onset of the disease, and patients can either improve or deteriorate into a more severe case. Type $\mathrm{H}$ patients (Fig. 2 ), approximately $20-30 \%$, behave more similarly to
COVID-19 patients with ARDS (“CARDS”), with low compliance, decreased gas volume due to edema, V/Q mismatch, and a possibility of lung recruitment. Considering the possibility of severe cases, respiratory support should be given to those with signs of respiratory failure

Considering the possibility of severe cases, respiratory support should be given to those with signs of respiratory failure such as $\mathrm{SpO} 2 \leq 93 \%$, increased work of breathing and tachypnea (for age), low $\mathrm{PaO} 2 /$ FiO2 ratio, high oxygenation index (OI) or high oxygen saturation index $(\mathrm{OSI})^{3}$.

Given the paucity of information regarding respiratory support of pediatric patients with Sars-CoV-2, we believe these recommendations may guide the ventilatory therapy in those children.

\section{NONINVASIVE RESPIRATORY SUPPORT}

Nasal prongs/reservoir mask (Low flow $<4 \mathrm{~L} / \mathrm{min}$ ): Should only be used in mild respiratory illness, with no respiratory distress ${ }^{4}$, since they do not generate recruitment of the lungs. If used, it may improve oxygenation but not the $\mathrm{PaO} 2 / \mathrm{FiO} 2$ ratio and may delay 
other ventilatory therapies ${ }^{3}$.

High flow nasal cannula (HFNC): May be used in cases with no severe hypoxemia. Response to treatment has to be assessed within 30-60 minutes from the initiation of therapy. If no response (SpO2/FiO2 < 220 or $\mathrm{FiO} 2>0,4$ for $\mathrm{SpO} 2>92 \%)^{5}$, other respiratory support should be considered. This therapy generates aerosol $^{6}$ and should never be used if there is no appropriate airborne isolation (negative pressure and $>12$ air changes per hour) and adequate personal protective equipment (PPE) $)^{4,6}$.

Continuous positive airway pressure (CPAP)/ Bilevel positive airway pressure (BiPAP): Can be used in moderate cases, if SatO2/FiO2 is between 221-264. A trial can be done during 60-90 minutes and response to treatment has to be assessed. If no response $(\mathrm{SpO} 2<92 \%$ and/or $\mathrm{FiO} 2>0,6)$ tracheal intubation is recommended ${ }^{5}$. Like in HFNC, this kind of support is also aerosol-generating and should only be used if appropriate precautions are available. A helmet is the preferable interface ${ }^{8}$ (watch out for risk of self-inflicted lung injury), if not available, a full face mask is the second choice. There can be no leaks during its use and it is necessary to use an adequate filter (HEPA or HMF).

If respiratory drive does not decrease with oxygen supply and use of non-invasive support with the child presenting significant spontaneous inspiratory efforts, tissue stress and increased pulmonary transvascular pressures will occur, with progressive worsening of lung function. To aim for an adequate gas exchange, avoiding P-SILI - patient self-inflicted lung injury.
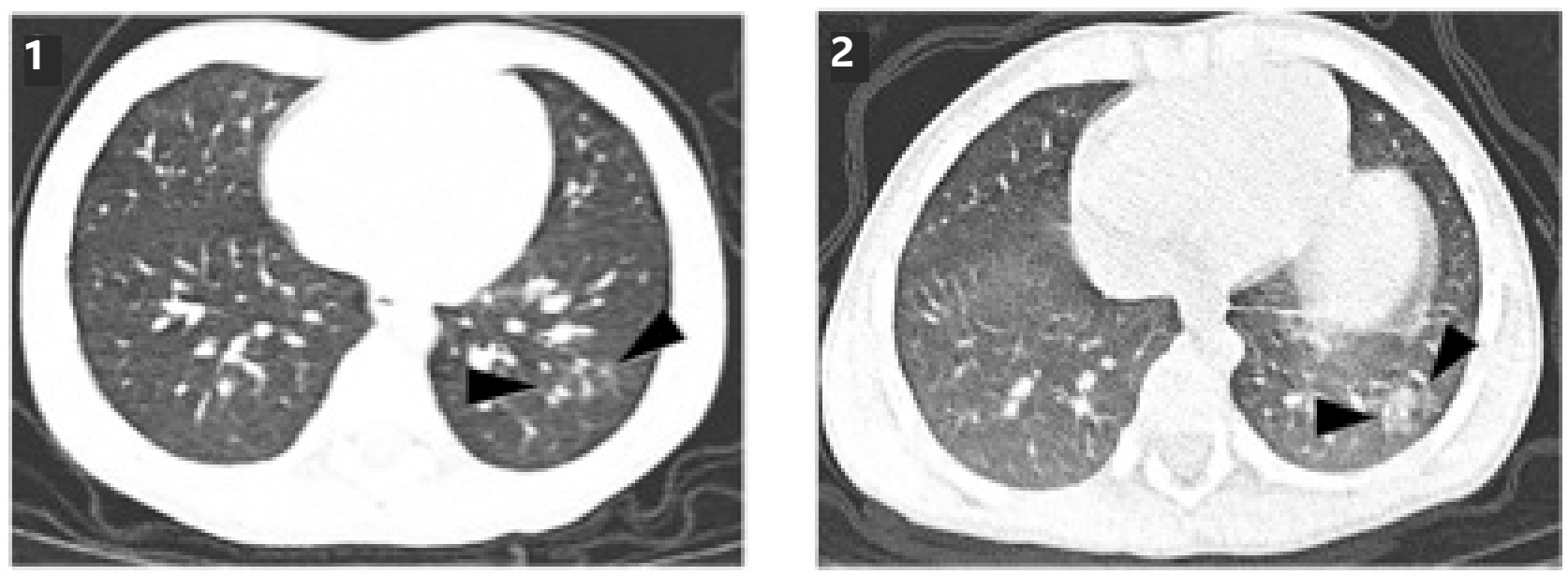

\section{AIRWAY MANAGEMENT}

Intubation is a critical procedure for patients with COVID-19 and also for healthcare workers. It has a high risk of viral shedding, so when it is performed, some important precautions are suggested ${ }^{9,10}$ :

- Preferably negative pressure ventilation rooms with ante-room to minimize exposure to aerosol or droplets

- Adequate PPE: Impervious gown, theatre hat, gloves, N95 facial mask, protection goggles, face shield ${ }^{11}$

- Disposable equipment is preferred over reusable equipment

- Closed-circuit aspiration

- Pre-oxygenation should be performed using a occlusive face mask with a viral filter (HEPA or HMF) or maintain the previously used device

- Avoid bag-mask ventilation if possible

- Intubation should be performed by the most experienced professional available and there must be a limited number of healthcare professionals to avoid exposure

- Use rapid sequence intubation to minimize exposure to droplets and aerosol: we suggest using fentanyl(1-2mcg/kg), midazolam (0,1-0,2mg/ $\mathrm{kg})$, and rocuronium (1-2mg/kg). Use atropine $(0,02 \mathrm{mg} / \mathrm{kg})$ in younger infants.

- Video laryngoscope is preferable but should be used by airway operators who are proficient in their use.

- Use cuffed cannulas (inflate the tracheal tube cuff up to $20 \mathrm{cmH} 2 \mathrm{O}$ initially). We suggest occluding the cannula with a clamp to avoid exposure to droplets and aerosol.

FIGURE 1. Patchy ground-glass opacification (arrowheads).

FIGURE 2. Also ground-glass but with more severe opacifications and edema. Adapt. from Wei Li, Huaqian Cui, Kunwei $\mathrm{Li}$, Yijie Fang, Shaolin Li. Chest computed tomography in children with COVID-19 respiratory infection. Pediatric Radiology. doi: 10.007/soo247-020-04656-7 


\section{INVASIVE RESPIRATORY SUPPORT}

Mechanical ventilation is essential when it comes do COVID-19 treatment, and it seems that the hypoxemic respiratory failure resulting from COVID-19 may be different from usual types of acute respiratory distress syndrome (ARDS) ${ }^{14}$. As we described, there seem to be two different types of pulmonary injuries (there are other described patterns of lung injury that determine a rationalization for the use of different pulmonary protection strategies in relation to the different stages) and they require different ventilation strategies. We recommend compliance evaluation at least twice a day, using electric impedance tomography (EIT) without perfusion analysis, if available, or performing respiratory mechanics to determine which type of pulmonary pattern seems more predominant.

For type L patients (high compliance), we suggest using PEEP around $8 \mathrm{cmH} 2 \mathrm{O}$ for infants, toddlers, and preschoolers, and PEEP around 10cmH2O for children older than 6 years old. Tidal volume should be around $6-8 \mathrm{ml} / \mathrm{kg}$ of predicted body weight (PBW) $)^{15,16}$, and up to $8-9 \mathrm{ml} / \mathrm{kg}$ PBW may be tolerated. Inspiratory time should be age-appropriate with a decelerating flow. Plateau pressure (Pplateau) should be as low as possible, maintaining adequate PEEP and maximum driving pressure of $15 \mathrm{cmH} 2 \mathrm{O}$. Respiratory rate should be enough to keep $\mathrm{pH}>7,25$ and inspired oxygen fraction (FiO2) should be $<60 \%$. We do not recommend using recruitment maneuvers for this type of patient.

Type H patients (low compliance) are recommended to be treated with protective ventilation ${ }^{14}$. Maintaining 3-6ml $/ \mathrm{kg}$ PBW of tidal volume, initial PEEP should be around $10 \mathrm{cmH} 2 \mathrm{O}$ and titrated up to $12 \mathrm{cmH} 2 \mathrm{O}$ (similarly to low PEEP table from ARDS network ${ }^{17}$ ), keeping in mind that excessive fluids may influence in inadequate PEEP titration. Plateau pressure should be $<30 \mathrm{cmH} 2 \mathrm{O}$, FiO2 $<60 \%$, inspiratory time should be age-appropriate with a decelerating flow, the respiratory rate should be enough to keep $\mathrm{pH}>7,25$, pCO2 should be monitored by capnography (preferably volumetric capnography) and we recommend using permissive hypercapnia $(\mathrm{pH}>7,2)$. Goal $\mathrm{SpO} 2$ is $93-96 \%$, as supraphysiologic arterial oxygen can be associated with higher mortality ${ }^{18}$. Regarding ventilatory modes, we suggest using pressure-controlled modes.

Some patients may present with wheezing or some kind of lower airway obstruction, and they should be treated with bronchodilators (preferably dosimetric or spray inhalers) and magnesium sulfate if necessary (initial dosing of $50 \mathrm{mg} / \mathrm{kg}$, infusion during at least 30 minutes). We recommend using low respiratory frequencies, inspiratory/expiratory time ratio of 1:3-1:4, and the lowest PEEP possible, avoiding auto-PEEP.

\section{ANALGESIA, SEDATION AND NEUROMUSCULAR BLOCKING AGENTS}

Appropriate administration of analgesia and sedation is an essential component in the care of mechanically ventilated patients. The promotion of comfort and lowering of anxiety, fear, and anguish is part of the routine. However, the use of excessive dosage or the fear of undersedation is a difficulty in the management of these patients.

The use of excessive of sedation indicated a strong association with poor outcomes, including a longer period of immobilization, more weakness, sleepwake disturbance and delirium, increase in time of mechanical ventilation and hospital length of stay, higher mortality, cognitive decline, and psychological complications. ${ }^{18,19}$

Recent studies and the last adult (2018) Guideline - Clinical Practice Guidelines for the Prevention and Management of Pain, Agitation/Sedation, Delirium, Immobility, and Sleep Disruption in Adult Patients in the ICU- shows that light sedation should be achieved to make the patient comfortable, calm and cooperative, resulting in an improvement of all poor outcomes described above and facilitating patient-ventilator interactions. ${ }^{19-21}$

All sedation strategies should start by assessing and reaching adequate pain control; the priority is effective analgesia and, consequently, a calm patient. To achieve good management of analgesia and sedation, nowadays, the use of scales (grade of recommendation $\mathrm{A}$ ) is recommended. The pain scale recommended and appropriate is the FLACC. The name FLACC is an acronym of five categories: Face, Legs, Activity, Cry, Consolability. Each category scores 0 to 2 points, totaling 10 points. The resulting scores correlate with the degree of pain: 0 for no pain or comfortable; 1-3 for mild pain; 4-6 for moderate pain; and 7-10 for intense pain. It can be used in many age groups, clinical settings, and cognitive states. ${ }^{19,20,21,23}$

In the initial management, analgesia may be the priority to achieve adequate pain control and level 


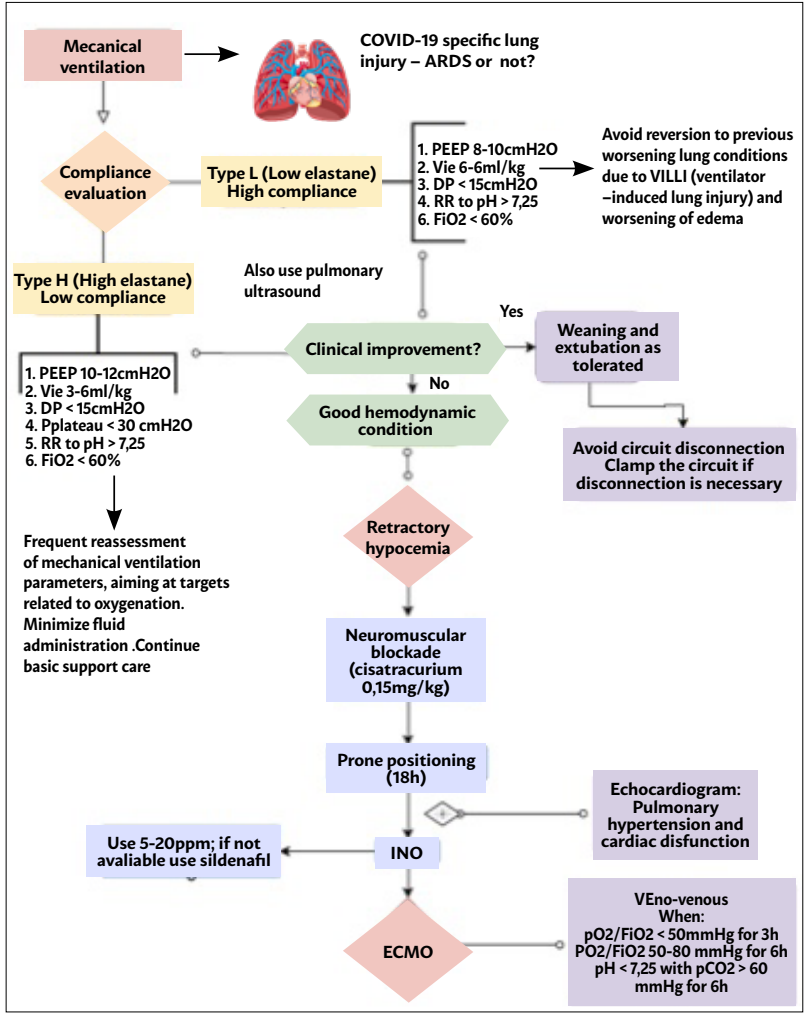

FIGURE 3. Mechanical ventilation management flowchart in critically ill pediatric patients

of sedation. So, the use of a strong opioid is recommended, being fentanyl the preferred. Fentanyl can be initiated intermittent and titrated to a continuous infusion to achieve adequate analgesia, guided by the FLACC scale. ${ }^{19-22}$

In the past, the primary sedation strategy in mechanically ventilated patients was deep sedation; however, a light level of sedation with an analgesia-first approach is recommended currently. The administration of sedation should only be considered after achieving adequate analgesia. As is the case with analgesia, bedside scales may be used to avoid deep sedation, prolonged ventilation, longer ICU and hospital stay, delirium, and weakness. The ESPNIC recommendation is the COMFORT Behavior scale (level A evidence). The COMFORT-B scale aggregates six behavioral parameters, in which 1 indicates deeper sedation and 5 more agitation for each parameter. Their sum should guide the titration of sedative drugs (<11: oversedation; 11-22: adequate sedation; $>22$ : undersedation). ${ }^{20,23,24}$ The choice of the ideal sedative agent is difficult and must take into account the pharmacology of the drugs and the different pathologies. The most frequently used sedatives in the pediatric ICU are benzodiazepines - midazolam. The initial use of the sedative drug must be after adequate analgesia in acute respiratory distress to help with patient management and avoid patient-ventilator asynchrony. However, it is still important to keep using scales to avoid unnecessary deep sedation, resulting in less delirium, shorter duration of mechanical ventilation, and improved mortality. ${ }^{20,21,22}$

With the use of continuous fentanyl and midazolam, it is important to consider the association of another drug. The emerging pediatric literature suggests the benefits of dexmedetomidine, an $\alpha$ - 2 agonist, improving outcomes. ${ }^{24}$ Dexmedetomidine has both analgesic and sedative effects, and, unlike other sedatives, does not depress the respiratory drive. Multiple clinical trials have demonstrated its efficacy and safety when used for longer-term sedation in the ICU. The 2018 PADIS guidelines recommend dexmedetomidine for sedation over benzodiazepines. ${ }^{21,22}$ In cases associated with asthma, consider the association of ketamine with fentanyl and midazolam.

However, several management aspects of acute respiratory distress can make light-sedation strategies (as described) challenging, including low tidal volume ventilation, high positive end-expiratory pressure (PEEP), prone and lateral positioning, and the patient evolving with refractory hypoxemia. On this occasion, consider the need for deeper sedation associated with neuromuscular blockade (NMB). This strategy may help improve hypoxemia by decreasing inflammation, decreasing oxygen consumption, increasing alveolar recruitment, improving patient-ventilator synchrony, and improving oxygenation by preventing the contraction of respiratory muscles when cisatracurium is administered in the first 48 hours. ${ }^{22,25,26}$ However, another study showed that among those who were treated with a higher PEEP strategy, the administration of continuous cisatracurium did not result in significantly lower mortality at 90 days. ${ }^{27}$ Therefore, we recommend, in cases of refractory hypoxemia, to associate intermittent BNM, which can improve oxygenation with lower barotrauma and without ICU weakness. In view of all of the above, consider, if possible, the use of Bispectral Index (BIS) to titrate sedative doses within BNM infusion, to ensure an adequate level of sedation. ${ }^{5,8,29}$

\section{REFRACTORY HYPOXEMIA}

A patient can be considered to have refractory hypoxemia when $\mathrm{SpO} 2<90 \%$, FiO2 $>60 \%$, driving pressure $>15 \mathrm{cmH} 2 \mathrm{O}$, Pplateau $>30 \mathrm{cmH} 2 \mathrm{O}$, and 
pCO2 has no response to ventilation and dead space reduction. If they have some of those features we recommend using the following strategies:

- Prone position: It is known that prone position decreases the V/Q mismatch due to pulmonary shunting $^{14}$ and optimizes ventilation. We suggest a trial and, if there is good response (pO2 increases more than 20mmHg, $\mathrm{SpO} 2>93 \%$, improvement in EIT and ventilation), maintain it for $18 \mathrm{~h}$ with $6 \mathrm{~h}$ intervals (if high workload, the patient can be kept in the prone position for up to $24 \mathrm{~h}$ ), and repeat if necessary. If the use of the prone position is not possible, the patient should be lateralized, keeping the more affected lung upwards.

- Nitric oxide: A potent pulmonary vasodilator, inhaled nitric oxide was used as rescue therapy in patients with refractory hypoxemia because it enhances pulmonary perfusion to areas with better ventilation; in the same way, it can be associated in patients with Sars-Cov-2 pulmonary thrombosis. It may show a transient improvement in $\mathrm{PaO} 2 / \mathrm{FiO} 2$ ratio. We suggest an initial dosing of 5 parts per million (ppm) to 20ppm. If nitric oxide is not available, we suggest using sildenafil (0.5-2 mg/kg/dose, every 4-6 hours with a maximum of $20 \mathrm{mg} /$ dose, every 8 hours).

- Recruitment maneuvers: We do not recommend the routine use of recruitment maneuvers. They can be performed if there is adequate monitoring, expertise, and clinical condition - we suggest a trial of step-by-step titration of PEEP. Beware of mechanical complications such as barotrauma and hemodynamic instability.

- High-frequency oscillatory ventilation (HFOV): We recommend against its use due to the risk of contamination of healthcare workers and other caregivers. It may be used in units with adequate isolation.
- Extracorporeal membrane oxygenation (ECMO): Consider veno-venous ECMO if in an experienced center, when the patient maintains $\mathrm{pO} 2 /$ $\mathrm{FiO} 2<50$ for $3 \mathrm{~h}$, or pO2/FiO2 $50-80$ for $6 \mathrm{~h}$, and $\mathrm{pH}<7,25$ with $\mathrm{pCO} 2>60$ for $6 \mathrm{~h}$, and when the patient doesn't meet the exclusion criteria (multiple organ dysfunction, severe neurologic disfunction, palliative care). ${ }^{30}$

\section{WEANING/ EXTUBATION}

Like it is with intubation, extubation is a critical moment in the care of critically ill patients with COVID-19. We recommend achieving very low ventilator settings before extubation $(\mathrm{FiO} 2<30 \%$, pressure support $\leq 10 \mathrm{cmH} 2 \mathrm{O}$ and PEEP $\leq 7 \mathrm{cmH} 2 \mathrm{O}$ ). We also suggest performing a spontaneous breathing test (SBT) with a T-piece for better evaluation of successful extubation. It is also important to test gag reflex and other airway protection reflexes prior to extubation as there have been reports of re-intubation in patients with aspiration or excessive airway secretions, some of them even requiring bronchoscopy ${ }^{31}$.

In pediatric patients, unplanned extubation is more frequent, so they should be closely monitored to minimize those events.

\section{CONCLUSION}

Although some cases of Sars-CoV-2 may behave similarly to ARDS, they have different pathological findings and should not be treated as the same illness. In addition, not every approach is equal. Children appear to have milder cases, but there have been some reports of severe cases ${ }^{32}$ that require mechanical ventilation. The suggested flowchart may contribute to the bedside management of severe pediatric cases of Sars-CoV-2 infection.

\section{REFERENCES}

1. Sun, Q., Qiu, H., Huang, M. et al. Lower mortality of COVID-19 by early recognition and intervention: experience from Jiangsu Province. Ann. Intensive Care 10, 33 (2020)

2. Gattinoni L. et al. COVID-19 pneumonia: different respiratory treatment for different phenotypes? (2020) Intensive Care Medicine; DOI: 10.1007/ s00134-020-06033-2

3. WeVent: Grupo Internacional de Ventilación Mecánica. Respiratory Management Protocol of Patients with Sars-CoV-2 (COVID-19). (2020)
4. Wax, R.S., Christian, M.D. Practical recommendations for critical care and anesthesiology teams caring for novel coronavirus (2019-nCoV) patients. Can J Anesth/J Can Anesth (2020). https://doi.org/10.1007/ s12630-020-01591-x

5. European Society of Paediatric and Neonatal Intensive Care: Practice recommendations for the management of children with suspected or proven COVID-19 infections from the Paediatric Mechanical Ventilation Consensus Conference (PEMVECC) and the section Respiratory Failure from the 
European Society for Paediatric and Neonatal Intensive Care (ESPNIC). (2020)

6. David S. H., Benny K. C., Thomas L., Owen T.Y. T., Fanny W. K., Susanna S. N., Tony G., Matthew T.V. C. European Respiratory Journal 2019 53: 1802339; DOI 10.1183/13993003.02339-2018

7. Wax, R.S., Christian, M.D. Practical recommendations for critical care and anesthesiology teams caring for novel coronavirus (2019-nCoV) patients. Can J Anesth/J Can Anesth (2020). https://doi.org/10.1007/ s12630-020-01591-x

8. Patel, B. K., Wolfe, K. S., Pohlman, A. S., Hall, J. B., \& Kress, J. P. (2016). Effect of noninvasive ventilation delivered by helmet vs face mask on the rate of endotracheal intubation in patients with acute respiratory distress syndrome: a randomized clinical trial. Jama, 315(22), 2435-2441.

9. Cheung, J. C. et al. Staff safety during emergency airway management for COVID-19 in Hong Kong. The Lancet Respiratory Medicine, 2020.

10. Brewster, D. J. et al. Consensus statement: Safe Airway Society principles of airway management and tracheal intubation specific to the COVID-19 adult patient group. Med J Aust, 2020

11. Ferioli, M. et al. Protecting healthcare workers from SARS-CoV-2 infection: practical indications. European Respiratory Review, v. 29, n. 155, 2020.

12. Poston, J. T.; PATEL, B. K.; DAVIS, A. M. Management of Critically III Adults With COVID-19. JAMA, 2020.

13. Gattinoni L. Preliminary Observations on the Respiratory Behavior. 2020;02(March):0-4.

14. The Pediatric Acute Lung Injury Consensus Conference Group: Pediatric acute respiratory distress syndrome: Consensus recommendations from the Pediatric Acute Lung Injury Consensus Conference. Pediatr Crit Care Med 2015; 16:428-439

15. Imber, David A.; Thomas, N. J.; Yehya, N. Association between tidal volumes adjusted for ideal body weight and outcomes in pediatric acute respiratory distress syndrome. Pediatric Critical Care Medicine, v. 20, n. 3, p. e145-e153, 2019

16. De Souza, L. CVF; Delgado, A. F.; De Carvalho, W. B. What Is the Best Weight to Obtain Protective Mechanical Ventilation?. Pediatric Critical Care Medicine, v. 20, n. 7, p. 693, 2019 .

17. National Heart, Lung, and Blood Institute ARDS Clinical Trials Network. Higher versus lower positive end-expiratory pressures in patients with the acute respiratory distress syndrome. New England Journal of Medicine, $v$. 351, n. 4, p. 327-336, 2004.

18. Palmer, E. et al. The Association between Supraphysiologic Arterial Oxygen Levels and Mortality in Critically III Patients. A Multicenter Observational Cohort Study. American Journal of Respiratory and Critical Care Medicine, v. 200, n. 11, p. 1373-1380, 2019.
19. Vincent JL, Shehabi Y, Walsh TS, Pandharipande PP, Ball JA, Spronk P et al: Comfort and patient-centred care wihout excessive sedation: the eCASH concept. Intensive Care Med 2016;42;962-971

20. Motta E, Luglio M, Delgado AF, Carvalho WB: Importance of the use of protocols for the management of analgesia and sedation in pediatric intensive care. Rev Assoc Bras 2016;62:602-609

21. Devlin JW, Skrobik Y, Gélinas C, Needham DM, Slooter AJC, Pandharipande PP, et al: Clinical Practice Guidelines for the Prevention and Management of Pain, Agitation/Sedation, Delirium, Immobility, and Sleep Disruption in Adult Patients in the ICU. Crit Care Med 2018;46 (9): e825-873

22. Pearson SD, Patel BK: Evolving targets for sedation during mechanical ventilation. Curr Opin Crit Care 2020;26: 47-52

23. Harris J, Ramelet AS, van Dijk M, Pokorna P, Wielenga J, Tume L, et al: Clinical recommendations of pain, sedation, withdrawal and delirium assessment in critically ill infants and children: an ESPNIC position statement for healthcare professionals. Intensive Care Med. 2016; 42: 972-986

24. Rosenberg L, Traube C: Sedation strategies in children with pediatric acute respiratory distress syndrome (PARDS). Ann Transl Med. 2019; 7 (19):509

25. Mefford B, Donaldson JC, Bissell BD: To Block or Not: Updates in Neuromuscular Blockade in Acute Respiratory Distress Syndrome. Annals of Pharmacotherapy. 2020:1-8

26. Papazian L, Forel JM, Gacouin A, Penot-Ragon C, Perrin G, Loundou A, et al: Neuromuscular Blockers in Early Acute Respiratory Distress Syndrome. N Engl J Med. 2010;363:1107-16

27. National Heart, Ling, and Blood institute PETAL Clinical Trials Network. Early Neuromuscular Blockade in the Acute Respiratory Distress Syndrome. N Engl J Med. 2019;380:1997-2008

28. Ho ATN, Patolia S, Guervilly C; Neuromuscular blockade in acute respiratory distress syndrome: a systematic review and meta-analysis of randomized controlled trials. Journal of Intensive Care. 2020;8:1

29. Bass S, Vancce ML, Reddy A, Bauer SR, Roach E, Torbic H, et al: Bispectral Index for Titrating Sedation in ARDS patients during Neuromuscular Blockade. American Journal of Critical Care. 2019;28:377-384.

30. Extracorporeal Life Support Guidance Document: ECMO for COVID-19 Patients with Severe Cardiopulmonary Failure. 2020.

31. Houzé, M. et al. Predictors of Extubation Failure Related to Aspiration and/ or Excessive Upper Airway Secretions. Respiratory Care, v. 65, n. 4, p. 475$481,2020$.

32. Lu, X. et al. SARS-CoV-2 infection in children. New England Journal of Medicine, 2020. 